\title{
Wave propagation in multiple flux tubes and chromospheric heating
}

\author{
S. S. Hasan ${ }^{1}$, A. van Ballegooijen ${ }^{2}$ and O. Steiner ${ }^{3}$ \\ ${ }^{1}$ Indian Institute of Astrophysics, Bangalore, India, \\ email: hasan@iiap.res.in \\ ${ }^{2}$ Harvard-Smithsonian Center for Astrophysics, Cambridge, U.S.A. \\ ${ }^{3}$ Kiepenheuer Institut für Sonnenphysik, Freiburg, Germany
}

\begin{abstract}
This investigation is a continuation of earlier work on the dynamics of the magnetic network. In a previous calculation (Hasan et al. 2005), we examined the response of a single flux tube to transverse motions of its footpoints. We now extend this analysis to a more realistic model of the network consisting of multiple flux tubes. We apply a transverse velocity perturbation uniformly along the lower boundary located at the base of the photosphere. Our 2-D MHD simulations enable us to study the complex wave pattern due to waves generated in the individual tubes as well as their interaction with those emanating from adjacent tubes. Our results show that the dominant heating of the chromosphere occurs due to slow magnetoacoustic waves in a region that is close to the central region of the flux tube.
\end{abstract}

Keywords. waves, MHD, Sun: atmosphere, Sun: photosphere, Sun: chromosphere, Sun: oscillations

\section{Introduction}

The present investigation is a continuation of our previous work (Hasan et al. 2005, henceforth Paper I) on wave propagation in the chromospheric network of the Sun. Paper I examined the response of a single flux tube due to transverse motions of its footpoints. We now extend the analysis to consider a more realistic model of the network consisting of three vertical flux tubes which have a filling factor of about $10 \%$ at the base of the photosphere in the network The tubes expand with height and merge with neighbouring tubes at a height of around $600 \mathrm{~km}$ above the photosphere. This study builds on earlier work on wave propagation in a 2-D stratified magnetized atmosphere by Rosenthal et al. (2002) and Bogdan et al. (2003).

\section{Model and Governing Equations}

We model a network element as a non-potential magnetic flux sheet with a width of $150 \mathrm{~km}$ at the base of the photosphere where $\beta=2$ on the symmetry axis (corresponding to a field strength of $1000 \mathrm{G}$ ), which decreases with height. The $\beta=1$ level on the axis occurs at a height of around $500 \mathrm{~km}$. Table 1 gives the model parameters on the axis.

Within the flux sheet, the pressure and density are lower than the corresponding values in the ambient medium, but the temperature is constant on horizontal planes. The magnetic field, density and pressure vary smoothly with distance from the axis. Details of the model are given in Hasan et al. (2005).

We consider wave generation in a network element by perturbing the lower boundary with a horizontal periodic motion of the form: $V_{z}(x, 0, t)=V_{0} \sin (2 \pi t / P)$ where $V_{0}$ is the amplitude of the motion and $P$ is the wave period. The simulation was carried out using 

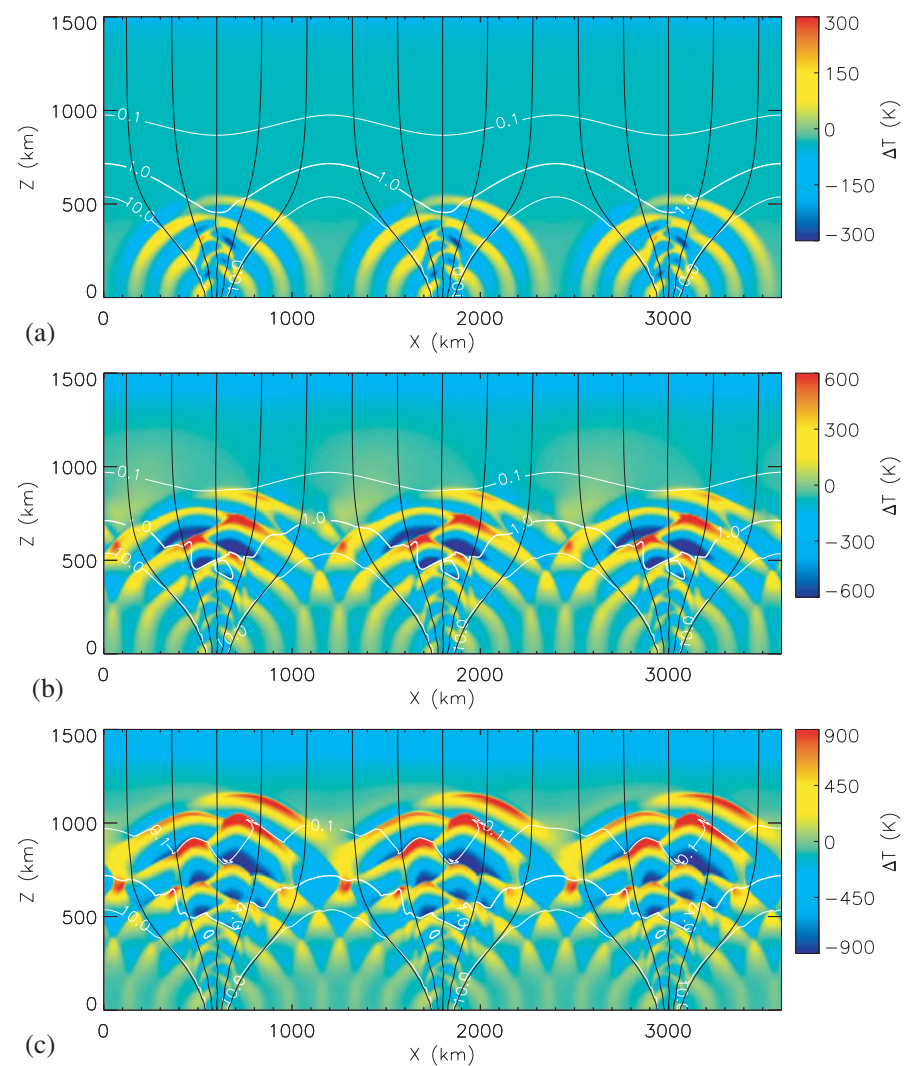

Figure 1. Temperature perturbation at (a) $75 \mathrm{~s}$, (b) $122 \mathrm{~s}$ and (c) $153 \mathrm{~s}$ in a network region consisting of 3 vertical flux tubes each of thickness $150 \mathrm{~km}$ at the base and a field strength on the axis of $1000 \mathrm{G}$. Wave excitation is due to a periodic horizontal motion at the lower boundary with an amplitude of $750 \mathrm{~m} \mathrm{~s}^{-1}$ and period of $24 \mathrm{~s}$. Black and white curves denote magnetic field lines and contours of constant $\beta=0.1,0.5,1.0$ (thick curve) and 10.

Table 1. Parameters on the Sheet Axis

\begin{tabular}{lll}
\hline \hline Variable & Base & Top \\
\hline Temperature & $4700 \mathrm{~K}$ & $8200 \mathrm{~K}$ \\
Sound speed & $7.1 \mathrm{~km} \mathrm{~s}^{-1}$ & $13 \mathrm{~km} \mathrm{~s}^{-1}$ \\
Alfvén speed & $5.5 \mathrm{~km} \mathrm{~s}^{-1}$ & $85 \mathrm{~km} \mathrm{~s}^{-1}$ \\
Magnetic field & $1000 \mathrm{G}$ & $44 \mathrm{G}$ \\
$\beta$ & 2.0 & 0.03 \\
\hline
\end{tabular}

the 2-D MHD code of Steiner et al. (1994) on a uniform grid of spacing $5 \mathrm{~km}$ (in both directions), with an upper boundary at $1500 \mathrm{~km}$ and a horizontal extension of $3600 \mathrm{~km}$. No flow boundary conditions were imposed on the lower and upper boundaries whereas periodic boundary conditions were used in the horizontal direction. More details can be found in Hasan et al. (2005). 


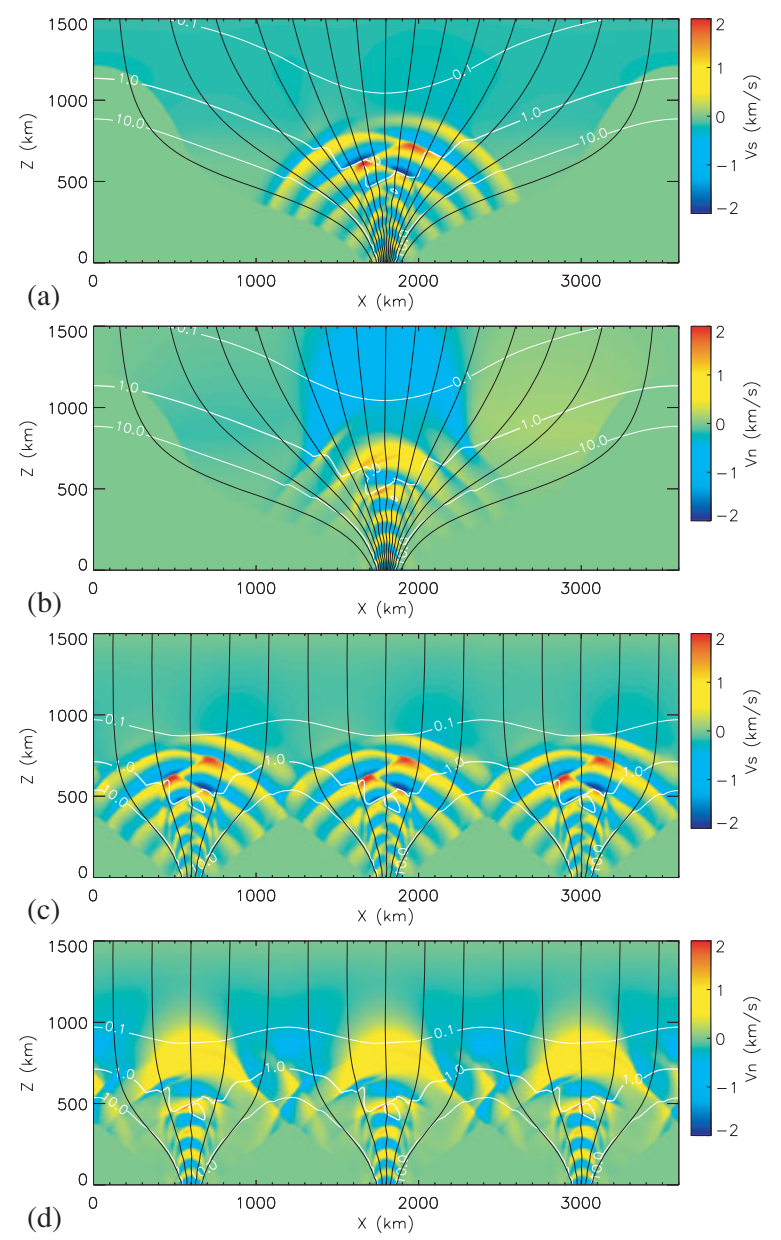

Figure 2. The field aligned $\left(V_{s}\right)$ and transverse $\left(V_{n}\right)$ components of the velocity in a single tube, (panels a - b) and multiple tubes (panels $\mathrm{c}-\mathrm{d}$ ) at $t=120 \mathrm{~s}$ due to periodic horizontal motion at the lower boundary, with an amplitude of $750 \mathrm{~m} \mathrm{~s}^{-1}$, and a period of $24 \mathrm{~s}$. The black and white curves denote the magnetic field lines, and contours of constant $\beta$ respectively. The velocity in the region corresponding to $B<20 \mathrm{G}$ is not shown.

\section{Dynamics of multiple flux sheets}

We examine the excitation of waves in a network patch consisting of three identical vertical flux tubes due to transverse driving of the lower boundary, with a period $P$ of $24 \mathrm{~s}$ and amplitude of $750 \mathrm{~m} \mathrm{~s}^{-1}$. Figures 1(a)-(c) show the temperature perturbation with respect to the initial value at each height at $75 \mathrm{~s}, 122 \mathrm{~s}$ and $153 \mathrm{~s}$. The black curves denote field lines and white curves depict contours of constant $\beta$ with values 0.1 (upper curve), 1.0 (thick curve) and 10 (lower curve).

At $t=75 \mathrm{~s}$, the waves in the individual tubes are sufficiently well separated from each other and are similar to the case of a single tube. However, at $t=122 \mathrm{~s}$ waves emanating from neighbouring tubes interact with each other especially in the ambient medium. The resulting interference produces a pattern that leads to a focusing of the wave front in the vertical direction. The wave pattern in any tube is not greatly affected by the presence of its neighbors. Furthermore, the acoustic-like waves, especially above the $\beta=1$ surface, are confined close to the central regions of the tubes, where they 
steepen and produce enhanced heating. This heating appears to be dominantly caused by the wave motions generated at the footpoints and not by the penetration of acoustic waves from the ambient medium or by waves coming from neighbouring tubes

Figures 2(a)-(b) and 2(c)-(d) show the field aligned $\left(V_{s}\right)$ and transverse $\left(V_{n}\right)$ components of the velocity for a single tube and multiple tubes respectively at $t=120 \mathrm{~s}$ (for the same parameters as before). In the weak field region $(\beta<<1)$, the anti-symmetric pattern (about the axis) in figures 2(a) and 2(c) can be identified with the longitudinal slow acoustic mode. On the other hand, the symmetric pattern seen as a uniform halo in figures 2(b) and 2(d) corresponds to the transverse MHD mode. There is a clear separation of the modes above the $\beta=1$ level where the fast mode speed increases rapidly with height and there is an increase in wavelength which manifests itself in the increase in the spacing of the colour contours. The shape of the contours is a consequence of the Alfvén speed being maximum on the axis (at a given height). Both cases for a single tube and multiple tubes clearly show the change in the flow pattern close to the $\beta=1$ level and the anti-symmetric structure of the acoustic waves away from the tube axis. Waves from neighbouring tubes interact with each other especially in the ambient medium. The resulting interference produces a pattern that leads to a focusing of the wave front in the vertical direction. The wave pattern in any tube is not greatly affected by the presence of its neighbors. Furthermore, the acoustic like waves, especially above the $\beta=1$ surface, are confined close to the central regions of the tubes, where they steepen and produce enhanced heating.

\section{Summary}

(a) We have identified an efficient mechanism for the generation of longitudinal motions from purely transverse motions at the base of tubes - similar results were found by James and Erdélyi (2002) for Alfvén waves in spicules;

(b) These longitudinal motions in the chromosphere are associated with slow magnetoacoustic waves that are responsible for heating the atmosphere which is localized to the regions close to the tube axis - a similar inference was made by De Pontieu et al. (2004) in a different context;

(c) We find that the main contribution to the heating comes from motions generated within the tube and not from the ambient medium or from waves from neighbouring tubes.

\section{Acknowledgements}

SSH and OS are grateful for support by grants from the Department of Science and Technology, Government of India (grant DST/INT/DAAD/P246/2006) and the German Academic Exchange Service (DAAD grant D/05/57/687).

\section{References}

Bogdan, T.J. et al. Carlsson, M. Hansteen, V., McMurry, A., Rosenthal, C. S. et al. 2003, ApJ, 599,626

De Pontieu, B., Erdélyi, R., James, S.P. 2004, Nature, 430, 536

Hasan, S.S., Kalkofen, W., van Ballegoiijen, A.A. Steiner, O. 2005, ApJ, 631, 1270 (Paper 1)

James, S.P., Erdélyi, R. 2002, Astron. Astrophys., 393, L11

Rosenthal, C.S., Bogdan, T. J., Carlsson, M., Dorch, S. B. F., Hansteen et al. 2002, ApJ, 564, 508

Steiner, O., Knölker, M. \& Schüssler, M. 1994, in: R. J. Rutten \& C. J. Schrijver (eds.), Solar Surface Magnetism, NATO ASI Ser. C-433 (Dordrecht: Kluwer), 441 